\title{
Knotted globular ring polymers: how topology affects statistics and thermodynamics
}

\author{
M. Baiesi, ${ }^{*, \dagger, *}$ E. Orlandini, ${ }^{\dagger, \dagger}$ and A. L. Stella ${ }^{\dagger,+}$ \\ Department of Physics and Astronomy, University of Padua, Via Marzolo 8, I-35131 Padova, \\ Italy, and INFN, Sezione di Padova, Via Marzolo 8, I-35131 Padova, Italy \\ E-mail: baiesi@pd.infn.it
}

\section{Abstract}

The statistical mechanics of a long knotted collapsed polymer is determined by a free-energy with a knot-dependent subleading term, which is linked to the length of the shortest polymer that can hold such knot. The only other parameter depending on the knot kind is an amplitude such that relative probabilities of knots do not vary with the temperature $T$, in the limit of long chains. We arrive at this conclusion by simulating interacting self-avoiding rings at low $T$ on the cubic lattice, both with unrestricted topology and with setups where the globule is divided by a slip link in two loops (preserving their topology) which compete for the chain length, either in contact or separated by a wall as for translocation through a membrane pore. These findings suggest that in macromolecular environments there may be entropic forces with a purely topological origin, whence portions of polymers holding complex knots should tend to expand at the expense of significantly shrinking other topologically simpler portions. 1

\footnotetext{
${ }^{*}$ To whom correspondence should be addressed

$\dagger$ University of Padua

$\ddagger$ INFN, Sezione di Padova

${ }^{1}$ This document is the unedited Author's version of a Submitted Work that was subsequently accepted for publication in Macromolecules, copyright (c)American Chemical Society after peer review. To access the final edited and published work see DOI 10.1021/ma5020287
}

\section{Introduction}

A main goal of topological polymer statistics is that of determining up to what extent the thermodynamics of macromolecular chains is affected by the presence of a fixed topological entanglement. $1-26$ Clarifying issues related to knots and links in polymer physics has been since long recognized of key importance in a variety of fields, ranging from molecular biology to nanotechnology. $11+15[27-31$ So far, for isolated ring polymers, which are the object of the present study, most theoretical and numerical approaches provided informations referring to ensembles with unrestricted topology. This means that information on the frequency of occurrence of different knots is in general not available for models of random ring polymer configurations. One does not have an idea of the extent to which, at least for long chains, these frequencies could reveal universal features, possibly connected to topological invariants. Thus, it remains unclear how different topologies contribute to the global free energy of systems described in such ensembles. This situation contrasts with the fact that ring polymers are not expected to change their topology in most experimental situations. Another fundamental problem is that of understanding if the presence of a fixed topology can give rise to thermodynamic effects, which would not be revealed within mixed topology ensembles.

The difficulty of answering questions like those above and the complexity of the scenarios the answers outline, depend in first place on the degree of localization of topological entanglement in the 
conditions considered for the polymer. ${ }^{16}$ For example, when dealing with polymers in good solvent, we know that the prime components of a knot are weakly localized. ${ }^{17-19}$ Each prime component of the knot behaves almost like a small bead sliding along the backbone. This makes it relatively simple to guess the gross structure of finite size corrections to the free energy expected for a knotted ring. ${ }^{32}$ The form of these corrections and the behavior of asymptotic relative frequencies of different knots are now known in great detail thanks to studies based on samples of configurations of extremely long knotted self-avoiding rings on a lattice. ${ }^{32}$ Indeed, while as established also by rigorous theorems,, $37 / 38$ unknotted configurations in good solvent should occur with zero frequency in infinite chains, it is necessary to consider very long rings in order to observe with appreciable frequency knotted configurations. For swollen polymers the decay to zero with increasing backbone length of the frequency of initially dominant, unknotted configurations is too slow to allow a rich enough sampling of knots with short rings.

The situation is quite different, and in several aspects more interesting, in the case of globular ring polymers. For chains in bad solvent it is well established that topological entanglement is delocalized and spreads along the whole backbone. $1822-25$ This delocalization is accompanied by the fact that models for generating random polymer configurations show a rich spectrum of knots already for relatively short chains. The unknot in this case does not occur with zero probability relative to any knotted configuration in the limit of infinitely long rings. To the contrary, there is evidence of an asymptotic spectrum in which the relative frequencies of all knots with respect to the unknot approach finite, nonzero limits. $\stackrel{26}{ }$ Additional interest in the globuli is due to some recently discovered, ${ }^{25]}$ remarkable thermodynamic properties directly linked to topology, which manifest themselves in processes like translocation through membrane pores.

The first steps towards a characterization of the spectrum of knots in ring polymers in the globular phase were made in Ref. ${ }^{26}$ Due to the difficulty of sampling dense configurations by Monte Carlo, the investigation was limited to a single temperature below the $\Theta$ point of self-avoiding rings on cu- bic lattice with attractive nearest neighbor interactions. This study showed that the relative frequencies of different knots tend to finite asymptotic values and have a ranking consistent with a Zipf law. A further result concerns the dependence of the globular free energy on the topological invariants of the knot: while in the infinite ring limit the free energy per monomer is independent of topology, a dependence on the minimal crossing number $\left(n_{c}\right)$ of the knot enters in one of its subleading finite size corrections Ref. ${ }^{25 \mid 26}$ (an example of knot with $n_{c}=5$ is shown in Fig. 1). This last result suggests that the value of $n_{c}$ could play a relevant role in the thermodynamics of a knotted globule. A way to shed light on this role consists in studying the behavior of the globule under geometrical constraints which interfere with the topology. In Ref. ${ }^{25}$ this kind of investigation was first performed using slipping links which divide the single globule in two interacting and possibly knotted loops in equilibrium. The simulations showed that indeed the respective minimal number of crossings precisely determines the way in which the two loops share the total ring length on average. The findings of Ref. $\frac{25}{25}$ on the topological correction to the free energy per monomer were also the basis for explaining a remarkable and unexpected phenomenon occurring when the slip link is replaced by a hole in a repulsive plane separating the ring in two non-interacting globuli with different topologies. This configuration schematizes a ring polymer translocating through a membrane pore. The different minimal number of crossings of the two knotted loops induces an asymmetry in the free energies of the competing globuli favoring configurations in which the globule with more crossings keeps most of the available backbone length. This asymmetry thus distorts the two otherwise symmetrical global free energy minima due to the surface tension of the globuli.

While being of fundamental interest, the problems mentioned above should be directly relevant for practical applications. Besides the translocation processes, of central importance for biology, the study of topological spectra of DNA extracted from viral capsids is a typical context where the knowledge of spectra for specific models of random polymers is very valuable. ${ }^{11|12| 14 \mid}$ Model calculations like those carried out in the present work 


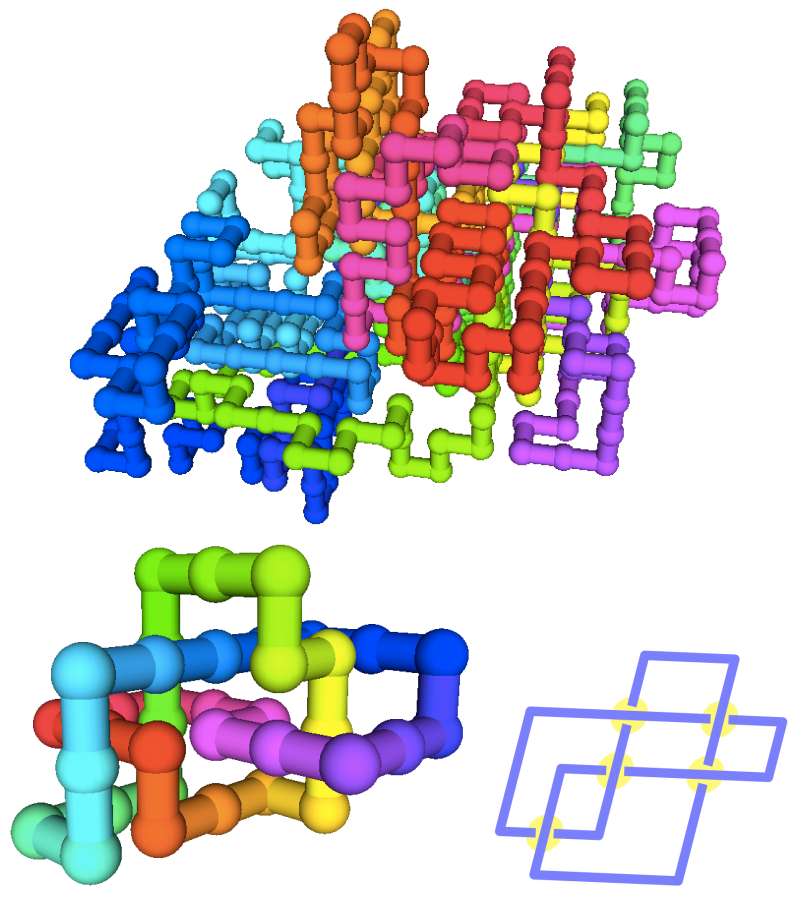

Figure 1: Example of a collapsed interacting selfavoiding ring with $N=500$ steps on the cubic lattice, and its shortened form after the $\mathrm{BFACF}^{39 / 40}$ reduction procedure with a low fugacity per step. The latter reveals that the ring holds a $5_{2}$ knot, whose projection with the minimal crossing number $n_{c}=5$ is also shown.

could also help in addressing issues like the known relative rarity of knots one finds in native globular proteins. ${ }^{41-43}$ The investigation of the effects of topological constraints on globular polymers, both in and out of equilibrium, is also expected to be a key to understand chromosomal architecture. ${ }^{44 / 45}$

In the present work we considerably extend and discuss in further detail some of the results presented in Refs. ${ }^{2526}$ Rather than assuming the more global perspective implicit in the Zipf law, here we look at the relative asymptotic frequencies of the simplest knots in globular ring polymers. The results furnish a clear evidence of the independence of temperature of these frequencies in the whole region below the $\Theta$ point and suggest the intriguing possibility that they represent universal, topology related numbers. This investigation proceeds in parallel with a better determination of the topological correction to the free energy of the globuli already postulated in. ${ }^{25}$ The analysis of data in a whole range of low temperatures gives stronger support to the conjectured form and corroborates the explanation of the already mentioned bias due to topology in the translocation of the globuli. Our analysis also shows that the strength of the topological correction is connected to the length of the knots in their ideal, minimal length form. $3100 / 46-48$ This connection sheds light on the reason why the correction itself seems to be determined primarily and almost exclusively by the minimal crossing number of the knots.

This article is organized as follows. In the next section we define the model and describe the methods of Monte Carlo simulation used for our analysis. Section Relative frequencies and free energies of knotted globuli is devoted to the analysis of the data for relative frequencies at different temperatures and to their extrapolation for infinitely long rings. The form of the finite size topological correction to the free energy and its dependence on both the minimal number of crossings and temperature are discussed in this section. In Section The role of $n_{c}$ in the thermodynamics of the globule we present results concerning the interference of geometrical constraints due to slip-links with topology. In Section Effects of the topological correction in translocation we refine our discussion of the effects of constraints due to translocation setups, and verify their consistency with the postulated topological finite size correction for the globular free energy. We also discuss the evidence of a connection between the topological correction and the properties of ideal knots. The last section is devoted to conclusions.

\section{Model and Simulations}

We model cyclic, flexible polymers with excluded volume as $N$-step self-avoiding rings on a cubic lattice (see Fig. 1). In order to induce collapse at low enough temperature $T$, we introduce an attractive interaction $(\varepsilon=-1)$ between nearest neighbors sites visited by the ring which are not consecutive along the backbone.

\section{Variable topology}

A first problem encountered in our analysis is that of generating a sufficient number of uncorrelated collapsed configurations by Monte Carlo. To this 
purpose we have to fix the temperature below the $\Theta$ point, which in our units is at $T_{\Theta} \simeq 3.72 .49050$ The pruned enriched Rosenbluth method (PERM) is efficient in generating collapsed polymer configurations; $; 26151$ as discussed below, we could obtain reasonably rich samplings down to $T=1.92$, for chains with length up to $N=1400$.

Of course, the study of the topological spectrum of the generated ring configurations involves another task, which is even more demanding: that of determining the knot type of each sampled configuration. This constitutes the bottleneck of our simulations. Indeed, it turns out to be a relatively minor problem the fact that PERM generates open chains and that we must discard most of them, keeping only those becoming rings upon addition of a further step.

Since the collapsed configurations are geometrically very intricated, with planar projections presenting huge numbers of crossings, before attempting a successful analysis of the knot type we need to simplify each configuration while keeping its topology unaltered. This simplification is achieved with a grand-canonical algorithm of the BFACF type, $39|40| 52$ which has the property of preserving the knot type because it lets the configurations evolve only with local and crankshaft deletion/insertion moves. In our case the simplification is achieved with a bias toward deletion that emerges from choosing a low fugacity $K$ per step. After a rapid shrinking of the ring (Fig. 1), it is eventually possible to analyze the knot type on the basis of HOMFLY polynomials within the "Knotscape" program. 53

\section{Fixed topology}

For studying the effects of external geometrical constraints on globular rings with fixed topology, we apply the BFACF grand canonical simulation method. As already explained in Ref., $\underline{25}$ there is a drawback of this method when applied to polymers below the $\Theta$ point: The length $N$ of the generated configurations does not grow continuously to $+\infty$ with the step fugacity, upon approaching its critical value from below. ${ }^{25}$ This behavior, consistent with the tricritical character of the $\Theta$ point, is due to the fact that the globule for $T<T_{\Theta}$ has an interfacial free energy growing as its surface, i.e. $\sim N^{2 / 3}$, in addition to the bulk one growing like $N$. As a consequence, it is not possible to gradually tune the length of the simulated rings just by varying $K$. In order to do so, as already explained in Ref., ${ }^{25}$ we introduce an extra $N$-dependent Gaussian weight multiplying the grand-canonical one for the configurations (an alternative method was recently proposed in ${ }^{54}$ ). This weight is maximum at a tunable $\bar{N}$ and has a sufficiently small width, so that $N \sim \bar{N}$ during the simulation. Thus, the sampling is essentially canonical up to relatively small fluctuations in $N$. This canonical character is needed in the analysis of how the competition between geometry and topology evolves as a function of the length of the rings, keeping the other parameters fixed.

\section{Relative frequencies and free en- ergies of knotted globuli}

The form of the free energy of a ring polymer at $T<T_{\Theta}$ can be guessed on the basis of an analogy with the swollen case and of the peculiar physical features of the globular phase. In particular, we can expect two facts: i) the free energy per monomer $f$ in the bulk of the globules is lower than the free energy $f_{s}$ of monomers on the interface between the globule and the solvent; ii) the number of monomers on the surface of a (smooth) globule scales asymptotically as $N^{2 / 3}$. Thus, a plausible starting ansatz ${ }^{26 \mid 55}$ for the canonical partition function of a collapsed ring with $N$ steps, in an ensemble with unrestricted topology, is

$$
Z_{N}(T) \simeq A e^{\kappa N} e^{\sigma N^{2 / 3}} N^{\alpha-2}
$$

where $\kappa=-\frac{f}{k_{B} T}>0$ and $\sigma N^{2 / 3}$ is a dimensionless interfacial correction to the bulk free energy. Indeed the number of monomers on the smooth globular surface grow as $N^{2 / 3}$, and $\sigma<0$. The factor $N^{\alpha-2}$ is just written in analogy with the swollen case and accounts for a possible further non extensive logarithmic correction to the free energy. In fact even tentative estimates of $\alpha$ for the globular phase were not available until quite recently, when some of the present authors proposed a Monte Carlo method for its determination. ${ }^{54}$ Here we focus on an ansatz for $Z_{k, N}$, the partition function of 
a globule with specific knot $k$. As a first hypothesis we could expect that at least some of the quantities in Eq. (1) assume a dependence on $k$ in order to represent the physics of a globule with fixed knot:

$$
Z_{k, N} \simeq A_{k} e^{\kappa_{k} N} e^{\sigma_{k} N^{2 / 3}} N^{\alpha_{k}-2}
$$

Studies of the self-avoiding rings at $T=\infty$, for which $\sigma=0$, have shown that in the swollen phase the amplitude $A_{k}$ indeed depends on $k$, while $\kappa_{k}$ does not seem to vary with the knot type ${ }^{32 / 33}$ For all temperatures $T>T_{\Theta}$ is also expected (and known exactly in the limit $T \rightarrow \infty$ 37) that $\kappa_{\emptyset}<\kappa$ where $k=\emptyset$ denotes the unknot. Remarkable in that case is the marked $k$ dependence of $\alpha$, which is increasing by one unit for each prime component of $k . \stackrel{3233}{3}$ Thus, the frequencies of different knots relative to the unknot turn out to diverge for $N \rightarrow \infty$ with a factor $N$ raised to a power equal to the number of prime components of $k$. This behavior as a function of $N$ is a strong confirmation that $\kappa_{k}$ does not depend on $k$ in that case.

\section{Knot frequencies}

We have sampled collapsed rings with the PERM algorithm at five different temperatures well below the $\Theta$ point: $1 / T=0.37,0.4,0.44,0.48$, 0.52 . The $N$ 's chosen for sampling are 400,500 , 600, 700, 800, 1000, 1200 and 1400. However, in some cases we will show only data up to $N=1000$ or $N=1200$ because the statistics (especially of some complex knots) was not rich enough for the larger $N$ 's at the lower temperatures.

When analyzing the globular phase, a first key property to study is the (absolute) probability $P_{\emptyset}$ of the unknotted configuration within the ensemble with unrestricted topology. As in the case of $T \rightarrow \infty$, the frequency of unknotted configurations is exponentially decreasing with $N$, but much more rapidly. As shown in Fig. 2, the decay constant $N_{\emptyset}$ is of the order of 400 for all $T$, while for selfavoiding polygons without attraction it would be of the order of 200000. This explains why simulations in the globular phase generate a much richer variety of knotted configurations than in the swollen phase already for moderately long chains. In Table 11 we list the estimated $N_{\emptyset}$ values for all temperatures. A trend of $N_{\emptyset}$ becoming lower with

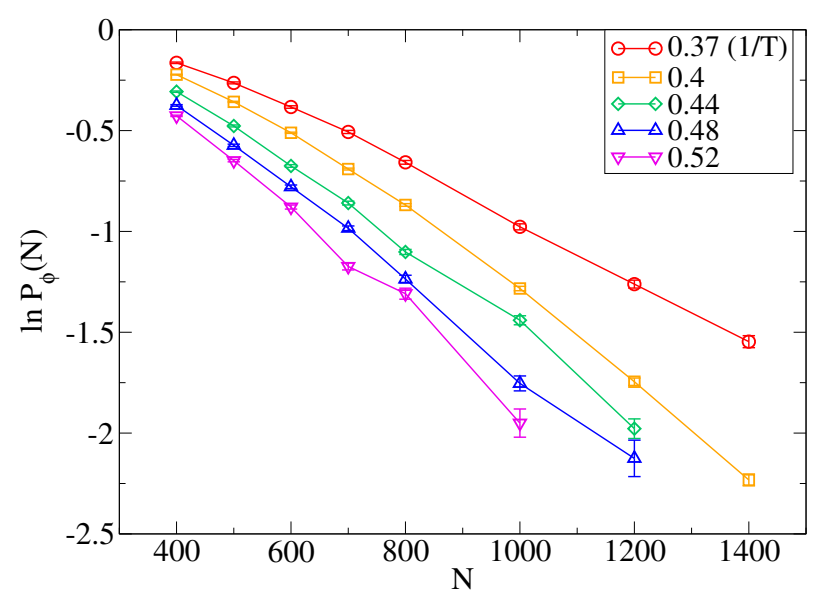

Figure 2: Logarithm of the frequency of unknot configurations as a function of $N$, for five different temperatures.

the temperature is evident. For $T \rightarrow 0$ one might expect that $N_{\emptyset}$ tends to a value similar or equal to the one estimated for Hamiltonian walks.

We made a systematic analysis of the frequencies $P_{k}$ of knots of increasing complexity, relative to that of the unknot. A first summary of our findings is provided in Fig. 3 where we plot the relative frequencies at $1 / T=0.48$ for the first few knots and for chain length ranging from $N=400$ up to $N=1000$. The plots report the logarithms of the frequencies as a function of $\frac{1}{N}$, and show two remarkable features: in all cases the extrapolation to $N \rightarrow \infty$ appears to give a finite limit; furthermore, the linearity of the plots suggests a correction $\sim 1 / N$ for the total free energy of the globules. The slope is approximately the same for different knots having the same minimal number of crossings, $n_{c}$. From the fact that the relative frequencies

Table 1: Some parameters for data at different temperatures: the $N_{\emptyset}$ for the exponential decay of the probability of unknots, the $C(T) \& a$ for fits $C(T) n_{c}^{a}$ of data in Fig. 5, and $C(T)$ of the fits $C(T) n_{c}^{1.33}$ shown in Fig. 5

\begin{tabular}{ccccc}
\hline $1 / T$ & $N_{\emptyset}$ & $C(T)$ & $a$ & $C(T)$ \\
\hline 0.37 & $678(14)$ & 242 & 1.30 & 223 \\
0.40 & $450(30)$ & 198 & 1.33 & 197 \\
0.44 & $460(45)$ & 171 & 1.33 & 170 \\
0.48 & $435(25)$ & 154 & 1.33 & 155 \\
0.52 & $380(40)$ & 138 & 1.36 & 145 \\
\hline
\end{tabular}




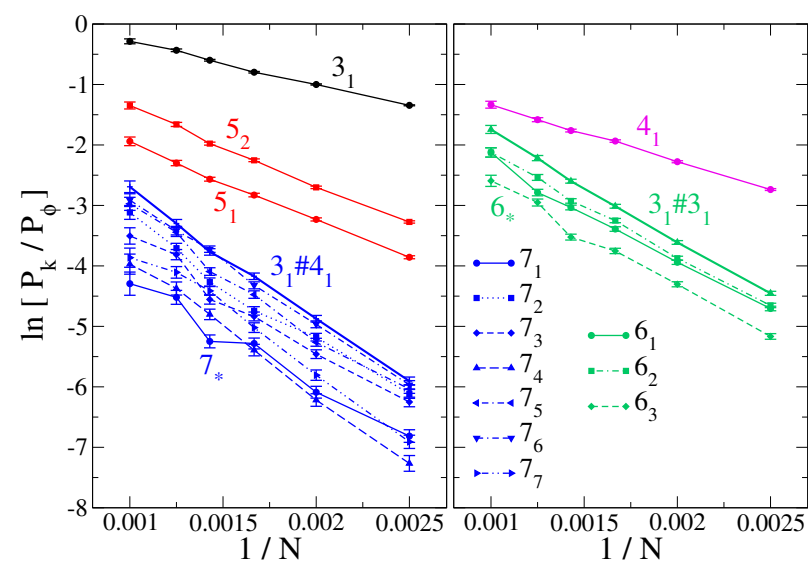

Figure 3: Frequency of knot types relative to the frequency of the unknot, at temperature $T=$ $1 / 0.48$ in the collapsed phase, as a function of $1 / N$. Colors distinguish knots according to their minimal crossing number. There are also two cases of composite knots (thick lines).

approach finite limits, we argue that, at the given temperature, if Eq. (2) applies, the parameters $\kappa_{k}$, $\sigma_{k}$ and $\alpha_{k}$ should be independent of $k$, while only the amplitudes $A_{k}$ should account for the different asymptotic frequencies. This could have been expected for the first two parameters, but marks an important difference from the swollen case as far as $\alpha_{k}$ is concerned. On the other hand, the $k$ dependence of $\alpha_{k}$ for $T \rightarrow \infty$ clearly originates from the localized character of prime knots, which does not hold in the globular phase.

Fig. 3 also suggests that there is a finite size correction to the free energy whose topology dependence is, at least to a good approximation, only through the minimal number of crossings of the knot $n_{c}$. The relative character of our frequency determinations does not in principle exclude that the part of globule free energy independent of topology could have a form different from that implied by Eq. (3) with $k$ independent parameters. In particular other topology independent finite size corrections could be present. In this work we focus on the topology dependent correction and on its consequences for the physics of the globule.

At this point it is important to check how the scenario provided by Fig. 3 changes upon varying the temperature of the globuli. At the same time one should determine as accurately as possible the dependence of the found topological free energy correction on both $n_{c}$ and $T$. In the first panel of
Fig. 4 we report, for five different temperatures below $T_{\Theta}$, the behavior with $1 / N$ of the topological free energy correction applying to the knot $3_{1}$.

A most remarkable feature in Fig. 4 is the fact that all the plots at different temperatures appear to extrapolate linearly to the same asymptotic relative frequency $\ln \left(A_{3_{1}} / A_{\emptyset}\right)$ for $1 / N \rightarrow 0$. Hence, the figure suggests that a fit of the form

$$
\ln \left(\frac{A_{3_{1}}}{A_{\emptyset}}\right)-M_{3_{1}}(T) \frac{1}{N}
$$

can be applied to all data, with the same asymptotic ratio and temperature-dependent slopes $M_{3_{1}}(T)$. The dashed lines are indeed a global fit of the data based on this assumption, confirming a nice consistency with it. The fact that the slope of the linear fits, $M_{3_{1}}$, decreases in modulus with temperature shows that the amplitude of the correction sensibly depends on temperature, contrary to previous expectations. 25

A finite, temperature-independent asymptotic relative frequency of a knot with respect to the unknot $\emptyset$ remains a valid assumption also for the knots $4_{1}, 5_{1}, 5_{2}, 61,33_{1} \# 3_{1}$ (see the panels of Fig. 4) and for all other simple knots we have considered (not shown). In the case of the two different knots with $n_{c}=5$ we see also that, while the asymptotic relative frequencies are different, the slopes of the linear plots at the same temperature are approximately the same. Thus, as already verified in the case of Fig. 3, the amplitude of the topological correction depends on temperature and, at least to a good approximation, on the minimal crossing number $n_{c}$ of the knot alone.

The asymptotic relative frequencies $\ln \left(A_{k} / A_{\emptyset}\right)$ extrapolated from the above plots are reported in Table 2. These frequencies are referring to knots which are delocalized into globules of increasing size. It is thus legitimate to expect that, besides being independent of temperature, they could be also universal with respect to the type of lattice in which the knotted configurations are realized. Remarkable universalities of quantities related to asymptotic amplitudes have been detected also in the study of the spectrum of knotted rings at $T \rightarrow$ $\infty$ phase. $\frac{35 / 36}{36}$ A challenge posed by Table 2 and its possible extensions concerns the verification of such universality and the possible link of the fre- 

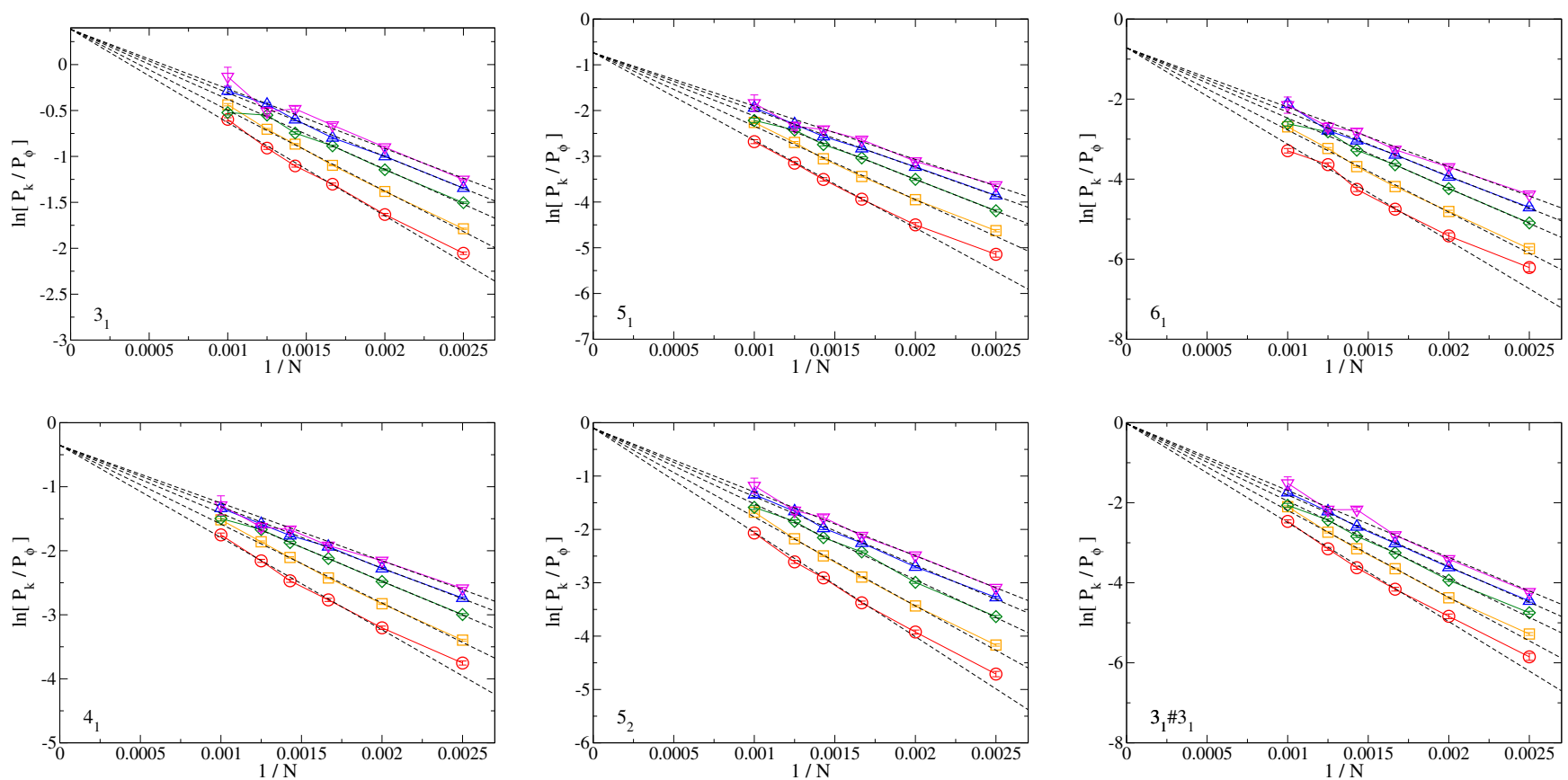

Figure 4: Probability of occurrence of a given knot type (6 cases shown, one for each panel) relative to the unknotting probability. In each panel 5 different temperatures are considered: $1 / T=0.37(\circ), 0.4(\square)$, $0.44(\diamond), 0.48(\triangle)$, and $0.52(\nabla)$.

quencies with topological invariants. For sure our data seem to exclude that, if the amplitude ratios depend on topological invariants, these could just reduce to $n_{c}$ alone. The spectrum of ring polymers in the globular phase is a remarkable case where topological polymer statistics puts all knots an a sort of equal footing, independent of their prime or composite character.

What one can efficiently extract from the data at finite $N$ reported in Figs. 3.4, is a tentative fitting form for the topological correction as a function of temperature and of $n_{c}$, assuming that these are the only parameters determining it. If in the various plots one assumes an asymptotic form as in Eq. (3), an expression of the form $M_{k}(T)=$ $C(T) n_{c}^{a}$, factorizing the dependences, seems reasonable for the correction amplitude, as already assumed in. ${ }^{25}$ This fitting form is applied globally in Fig. 5 to the data collected for different knots and different temperatures. The estimates, listed in Table 1, show that an optimal determination for the exponent is $a \simeq 1.33^{2}$. We have thus fitted again data with the one-parameter form $C(T) n_{c}^{1.33}$ and

\footnotetext{
${ }^{2}$ Previously $a \simeq 1.45$ was found. ${ }^{25}$ Moreover, we have discovered that the value of $T=2.5$ quoted in Ref. ${ }^{25}$ is not correct: simulations were actually run at $T=2$, which explains some discrepancies found in estimates of $C(T)$.
}

obtained similar values of $C(T)$, see Table 1 . In the inset of Fig. 5 we report the determinations of the amplitude $C(T)$ resulting from the last fits. Even if the temperature range covered by our determinations remains limited, there is evidence that the postulated $C(T)$ could vary linearly with temperature and could approach zero for $T \rightarrow 0$. An important fact are the relatively large values of $C$ determined for all temperatures considered. Such large values show that the topological correction is quite substantial for not too large globules. Notice that both $C(T)$ and the exponent $a$ are just intended to provide a convenient fitting for the quantities $M_{k}(T)$ reported in Table 2. In particular, one should not assign a particular meaning to the value of the exponent $a$.

\section{The role of $n_{c}$ in the thermody- namics of the globule}

Since globules in experiments are necessarily finite, the above findings about the topological correction to the globule free energy suggest that the invariant $n_{c}$ could play an important role in the thermodynamic behavior of collapsed knotted rings. In order to shed light on this role, a natu- 
Table 2: Estimates of the knot-dependent constants for some simple knots in globules. The column $M^{\prime}$ refers to parameters from fits of $\ln \left(P_{k} / P_{\emptyset}\right)$ vs. $1 / N$ only at $1 / T=0.48$, while the following columns are from fits assuming that all data at different $T$ converge to the same asymptotic value $\ln \left(A_{k} / A_{\emptyset}\right)$, as in Fig. 4. The last two columns contain the estimates of the typical globule length $\bar{l}$ for some knots for which we had clear peaks in the distributions $P\left(l_{1}\right)$ for the competition of Fig. 6(b). The column $\bar{l}$ shows direct estimates (positions of the peaks in the limit of long chain lengths, not shown), to be compared with predictions from Eq. (10) in the last column (we used $\sigma=-0.96(5)$, see Ref., 54 and values in the column $M(0.48))$.

\begin{tabular}{llllllllll}
\hline knot & $M^{\prime}(0.48)$ & $\ln \left(A_{k} / A_{\emptyset}\right)$ & $M(0.37)$ & $M(0.4)$ & $M(0.44)$ & $M(0.48)$ & $M(0.52)$ & $\bar{l}$ & Eq. (10) \\
\hline $3_{1}$ & $714(21)$ & $0.39(0.12)$ & $1020(73)$ & $885(71)$ & $765(59)$ & $696(57)$ & $652(54)$ & $49(1)$ & $66(11)$ \\
\hline $4_{1}$ & $929(15)$ & $-0.36(0.14)$ & $1434(89)$ & $1224(85)$ & $1055(72)$ & $953(68)$ & $897(64)$ & $67(2)$ & $80(12)$ \\
\hline $5_{1}$ & $1260(30)$ & $-0.74(0.18)$ & $1910(120)$ & $1600(120)$ & $1380(100)$ & $1250(90)$ & $1170(90)$ & $77(2)$ & $94(14)$ \\
$5_{2}$ & $1290(40)$ & $-0.10(0.17)$ & $1960(120)$ & $1670(110)$ & $1420(90)$ & $1280(90)$ & $1200(80)$ & $85(6)$ & $96(14)$ \\
\hline $6_{1}$ & $1660(80)$ & $-0.73(0.27)$ & $2400(190)$ & $2050(180)$ & $1750(150)$ & $1600(140)$ & $1480(130)$ & & \\
$6_{2}$ & $1690(40)$ & $-0.41(0.22)$ & $2570(160)$ & $2180(150)$ & $1880(130)$ & $1720(120)$ & $1570(110)$ & $105(6)$ & $114(16)$ \\
$6_{3}$ & $1795(85)$ & $-0.78(0.42)$ & $2700(300)$ & $2310(280)$ & $1970(240)$ & $1780(230)$ & $1630(210)$ & & \\
$3_{1} \# 3_{1}$ & $1805(35)$ & $0.00(0.26)$ & $2490(180)$ & $2190(170)$ & $1950(150)$ & $1800(140)$ & $1680(130)$ & & \\
\hline $7_{1}$ & & $-2.17(0.53)$ & $2980(390)$ & $2460(360)$ & $2110(320)$ & $1930(290)$ & $1830(270)$ & & \\
$7_{2}$ & & $-1.24(0.49)$ & $2980(360)$ & $2590(330)$ & $2210(290)$ & $2000(270)$ & $1890(250)$ & & \\
$7_{3}$ & & $-1.56(0.43)$ & $2920(320)$ & $2450(290)$ & $2110(250)$ & $1940(240)$ & $1800(210)$ & & \\
$7_{4}$ & $2260(60)$ & $-1.94(0.50)$ & $3040(350)$ & $2650(340)$ & $2310(300)$ & $2080(290)$ & $1890(250)$ & & \\
$7_{5}$ & & $-0.95(0.35)$ & $3050(250)$ & $2670(240)$ & $2300(210)$ & $2100(190)$ & $1980(180)$ & & \\
$7_{6}$ & & $-0.75(0.28)$ & $3160(200)$ & $2740(190)$ & $2350(170)$ & $2110(160)$ & $2000(140)$ & & \\
$7_{7}$ & & $-1.50(0.43)$ & $3150(310)$ & $2740(300)$ & $2350(260)$ & $2120(250)$ & $2030(220)$ & & \\
$3_{1} \# 4_{1}$ & $2110(55)$ & $-0.60(0.44)$ & $2960(320)$ & $2650(300)$ & $2310(260)$ & $2150(250)$ & $2000(220)$ & & \\
\hline
\end{tabular}

ral way is to apply to the globule geometrical constraints which can interfere with topology, and in particular with the topological invariant under examination. The response to such constraints could reveal interesting ways in which topology controls the behavior of the globule. A further possibility is that of considering geometrically constrained situations which allow a direct check of the presence and effects of the postulated free energy correction. In this section we pursue the first ways of analysis.

Since now we are interested in configurations with fixed topology, we use the BFACF method described in the previous section, and we tune the parameters to sample chains longer than those obtained by PERM for unrestricted topology. In this way, we arrive at lengths $N$ up to 7000 .

We first consider the effect of a slipping link on the statistics of the globule. The slipping link, sketched in Fig. 6(a) is such to divide the ring in two loops, of length $l_{1}$ and $l_{2}=N-l_{1}$. Each loop has a given knot, respectively $k_{1}$ and $k_{2}$, and the slipping link is narrow enough to prevent the translocation of the knots from one loop to the other. This is the interference between geometry and topology mentioned above. It should be stressed that the two loops are attractively interacting in this set up, so that they are forming a single globule.

It is interesting to analyze the fluctuations in the lengths of the two loops in equilibrium, and to verify in which way they possibly depend on the two knots in the loops. To this purpose we use the quasi-canonical simulation method preserving topology described in Section Relative frequencies and free energies of knotted globuli. In Fig. 7 we report the probability density function $P\left(l_{1} / N\right)$ of the fraction of total backbone taken on average by loop 1, for different $N$ and for different competing knots $k_{1}$ and $k_{2}$. All runs were rather extensive and performed at $T=2$, below the $\Theta$ point. In Fig. 7(a) we see the competition between two $3_{1}$ knots. The tendency of the distribution to become broader and flatter with increasing $N$ indicates that $l_{1}$ undergoes broad fluctuations, which grow proportional to $N$ itself. The same tendency 


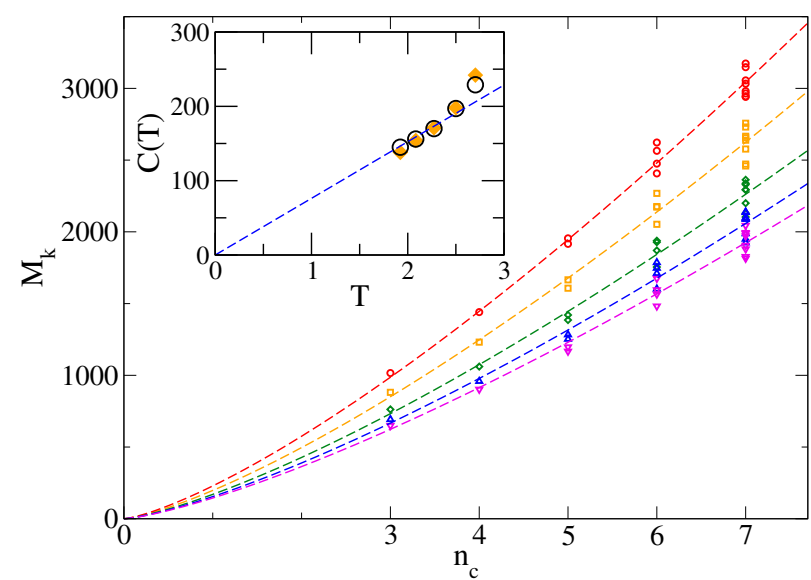

Figure 5: Estimates of $M_{k}$ for knots up to $n_{c}=7$, at five temperatures (see color/symbol code of previous figures). Note that data with the same $T$ and $n_{c}$ are quite well clustered. The dashed curves refer to fits of the form $\sim C(T) n_{c}^{1.33}$ (those in which $\sim C(T) n_{c}^{a}$, with $a$ being a parameter free to vary are similar). Inset: Estimates of $C$ as a function of temperature. Filled diamonds are values determined by not fixing the exponent $a$ in the fits, while empty circles refer to estimates obtained by fixing $a=1.33$ (i.e. the weighted average of the $a$ estimates obtained at the five different temperatures considered). The dashed line is a guide to the eye showing that $C(T) \sim T$ is indeed an acceptable ansatz. Fits of the most asymptotic black dots (not shown) give also asymptotic $T \rightarrow 0$ extrapolations to a value $C(0)$ consistent with zero.

is observed in other knot competitions displayed in Fig. 7. A case of 31 in loop 1 competing with $4_{1}$ in loop 2 is reported in Fig. 7. In this case there is an increasing skewness of the distribution for increasing $N$, showing that configurations in which the loop 1 is shorter than loop 2 are favored. This dominance occurs again in the presence of broad fluctuations, as indicated by the fact that the distribution broaden with increasing $N$. The distributions become more skewed in general if $n_{c 1}$ is much different from $n_{c 2}$, as one can see for a 31 vs. $7_{1}$ competition in Fig. 7 (d)], while they remain (approximately) symmetrical even for different knots if $n_{c 1}=n_{c 2}$ [case of a 61 vs. $3_{1} \# 3_{1}$ shown in Fig. 7(c)].

Given the reported increasingly broad distributions of loop lengths, it is not straightforward to predict the scaling of their averages $\left\langle l_{1}\right\rangle$ and $\left\langle l_{2}\right\rangle$

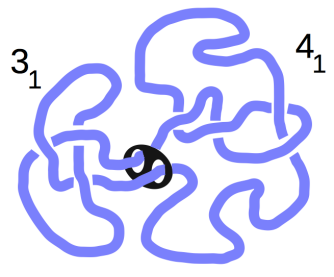

(a) (b)

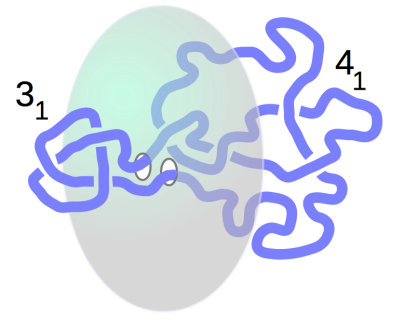

Figure 6: Sketch of a polymer ring with a slip-link separating two loops, the first holding a 31 knot and the second a $4_{1}$. Each knot is confined in its loop because the holes in the slip-link are narrow enough to prevent the passage of a blob containing more than one monomer. However, in this way the loop lengths may vary. In case (a) the two loops interact as a single globule, while in (b) they form two separate globules because the slip-link is in an impenetrable wall.

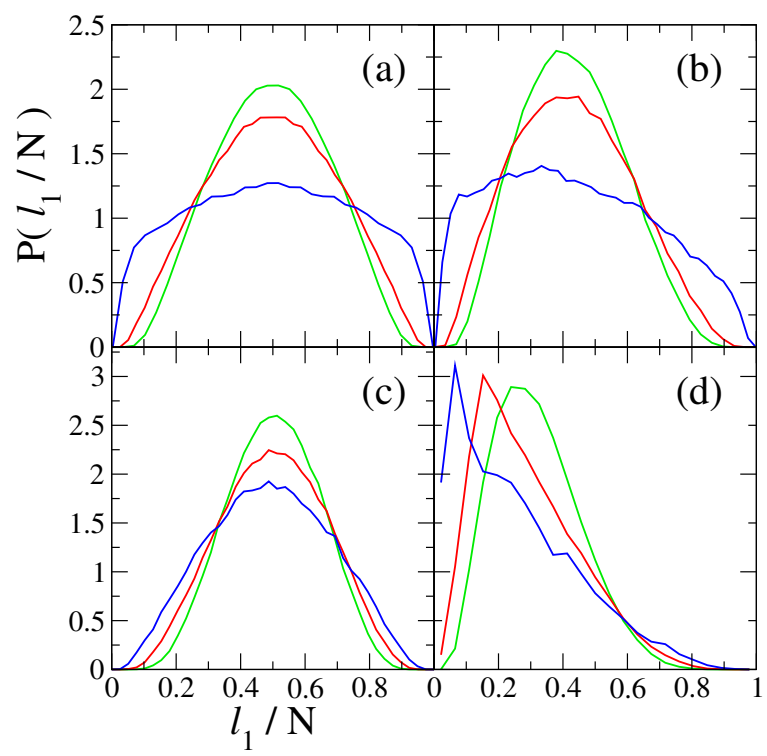

Figure 7: Probability distributions of the fraction of length of one of the two loops in a slip-link competition of knots [Fig. 6(a)], for different ring length: (a) $3_{1}$ vs. $3_{1}$, for $N=500$ (green), 1000 (red), 4500 (blue); (b) $3{ }_{1}$ vs. $4_{1}$, for $N=500,1000$, 5000; (c) $6_{1}$ vs. $33_{1} \# 3_{1}$, for $N=1000,2000,4000$; (d) $3_{1}$ vs. $7_{1}$, for $N=500,2000,7000$.

with $N$. However, the plots of $\left\langle l_{1}\right\rangle$ and $\left\langle l_{2}\right\rangle$ as a function of $N$ reported in Fig. 8 reveal a surprisingly simple linear behavior. For example, for the case $k_{1}=3_{1}$ vs $k_{2}=4_{1}$ in Fig. 8(a) we observe that $\left\langle l_{1}\right\rangle$ and $\left\langle l_{2}\right\rangle$ approximately grow proportional to $\frac{3}{7} N$ and $\frac{4}{7} N$, respectively (see the straight lines). 


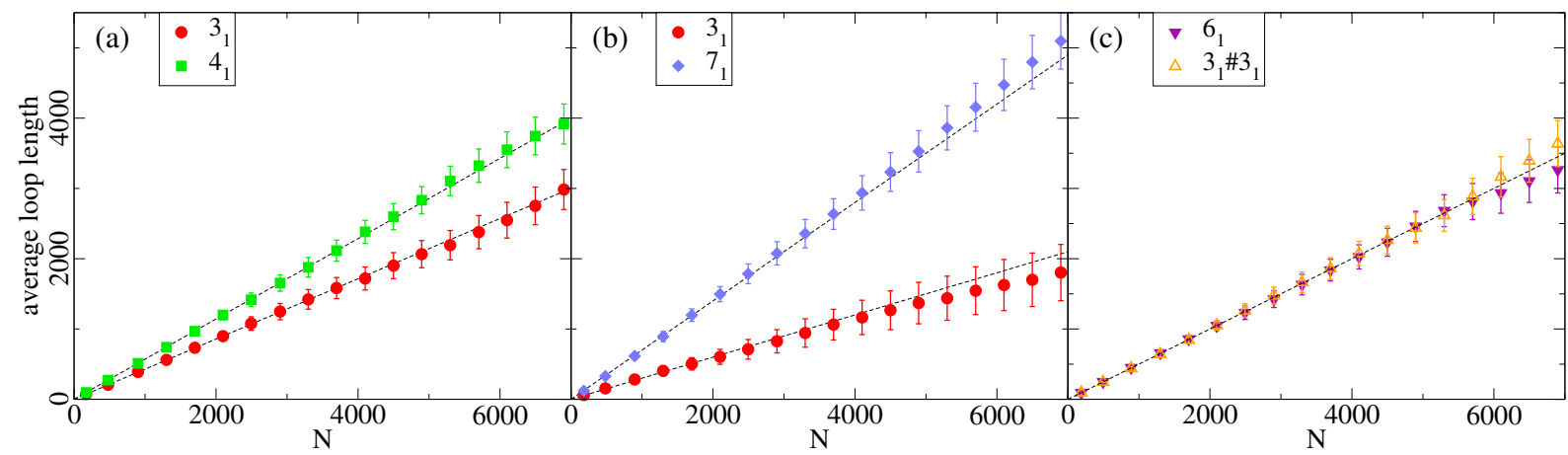

Figure 8: Average length of each of the two loops in which the globule is partitioned by the slip-link, see Fig. 6(a). The knots hosted by the two loops are (a) $33_{1}$ and $4_{1}$; (b) $3_{1}$ and $7_{1}$; (c) 61 and $3_{1} \# 3_{1}$. Straight lines have slopes predicted by Eq. (4).

This suggests that $n_{c}$ is the controlling parameter in the competition. Another example of competition is a $3{ }_{1}$ against 71 , as reported in Fig. 8(b). Here again we have seen that the fluctuations of $P\left(l_{1} / N\right)$ are broad, yet the average loop lengths are determined on the basis of the minimal crossing numbers of the competing knots, as $\frac{3}{10} \mathrm{~N}$ and $\frac{7}{10} \mathrm{~N}$. A further confirmation of the fact that $n_{c}$ alone determines the loop statistics, is given by the competition $3_{1}+3_{1}$ vs $6_{1}$. In this case [Fig. 8(c)] we see that the symmetry of $P\left(l_{1} / N\right)$ shown in Fig. 7(c) is also well reflected by the behavior of the average loop lengths $\left\langle l_{1}\right\rangle \simeq\left\langle l_{2}\right\rangle \simeq N / 2$.

On the basis of the above simulations one can postulate that the average loop lengths are determined according to

$$
\left\langle l_{i}\right\rangle=\frac{n_{c i}}{n_{c 1}+n_{c 2}} N
$$

where $n_{c i}$ is the minimal number of crossings in loop $i$. This is a deceptively simple law which could, however, be hardly guessed a priori. Of course, the provided evidence that this law applies is limited by simulation capabilities. Like in the case of the results presented in the previous section, there is the risk that the exclusive and sharp role postulated for $n_{c}$ is valid only to a very good approximation. For example, looking at the data for the $3{ }_{1} \# 3_{1}$ vs $6_{1}$ competition, the circumstance that the two loops are going to a fully symmetrical equilibrium for large $N$ can be reasonably guessed, rather than given for certain. On the other hand, even in the perspective of establishing approximate laws, the results presented here appear remarkable. Thinking to the enormous geometrical intricacy in which the essential topological crossings are hidden within the globule configurations, one would guess at first sight that the role of $n_{c}$ should not be so important.

\section{Effects of the topological correc- tion in translocation}

A way to make direct contact with the ansatz for the knotted globule free energy presented in the previous section is to replace the slipping link with an impenetrable wall presenting a hole through which the knotted ring is passing (Fig. 6). In this setting the two loops would be not interacting with each other, and would constitute independent knotted globuli just competing for backbone length, each one with its own surface exposed to the solvent. This resembles the translocation of a globular ring polymer through a membrane or a solid state nanopore (though in our setup the hole does not allow a full translocation, preserving the knots in each loop). Situations like this should be in principle within the reach of experimental investigation nowadays, thanks to progresses in nanophysics and nanotechnology.

Important here is the fact that the free energy of the whole system reduces to the sum of the free energies of the two globuli, due to their independence. Moreover, in the limit of one short loop, the complementary long loop acts just as a monomer reservoir. Below we show that $M_{k}$ is related to the typical chain length selected by the globule in that limit and to the length of ideal knots.

The logarithmic weight (or minus dimensionless 


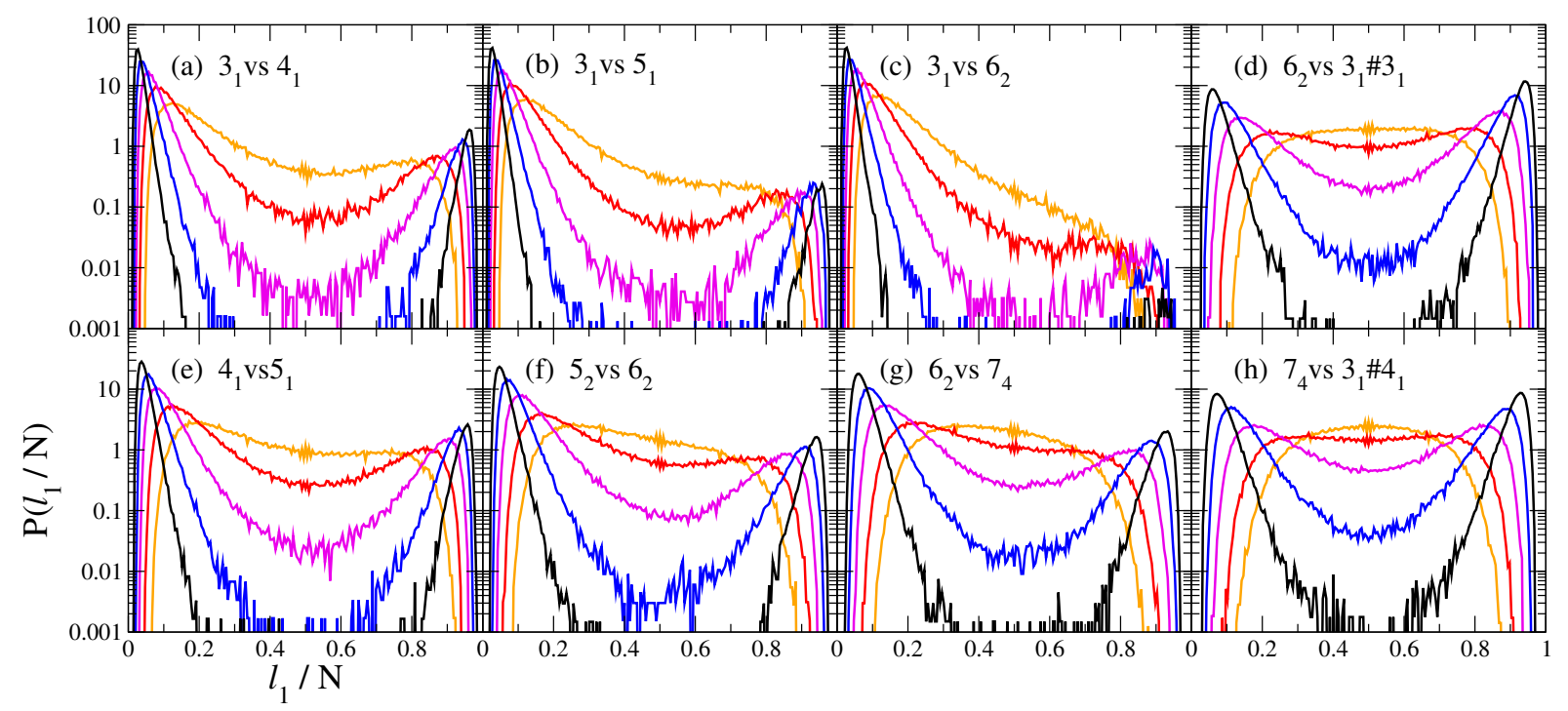

Figure 9: Probability distributions of the relative length of one of the two loops in a competition of two globular knots separated by a wall [Fig 6(b)], for different knot pairs. In each case five different $N$ values $\left(500,700,1000,1400\right.$, and 2000) are considered. The sequence (a)-(b)-(c) refers to the knot $3_{1}$ vs a progressively more complex knot: one can note an increasing unbalance in the distributions. The sequence (a)-(e)-(f)-(g) is for progressively more complex knots, showing that the minimum between the two maxima of the distributions occurs for longer $N$ 's if the hosted knots become more and more complex; this is due to a larger minimal knot size. Finally, (d) and (h) are examples of knots competing with other knots that have the same minimal number of crossings $n_{c}$ but are otherwise topologically different: the symmetry of the distributions confirms that $n_{c}$ is a good number to look at when knots are competing in the globular phase.

free energy) of the first globule of length $l_{1}$, defined as $\varphi_{k_{1}}\left(l_{1}\right) \equiv-F_{k_{1}}\left(l_{1}\right) / k_{B} T$ (where $F_{k_{1}}$ is the total loop free energy), is written as

$\varphi_{k_{1}}\left(l_{1}\right)=\ln A_{k}+\kappa l_{1}+\sigma l_{1}^{2 / 3}+(\alpha-2) \ln l_{1}-\frac{M_{k_{1}}}{l_{1}}$

where $\kappa, \sigma$, and $M_{k_{1}}$ are functions of $T$ (we are omitting further knot-independent corrections to scaling). Thus, for the translocating ring one can write the probability of the first loop length when competing with a knot $k_{2}$,

$$
P_{k_{1}, k_{2}}\left(l_{1}\right) \propto \exp \left[\varphi_{k_{1}}\left(l_{1}\right)+\varphi_{k_{2}}\left(N-l_{1}\right)\right]
$$

With the free energy form given in Eq. (5), in the case of $n_{c 1}=n_{c 2}$ one would expect $M_{k_{1}} \approx M_{k_{2}}$, i.e. a symmetric $P$, with two equivalent maxima determined by the surface tension term. This is what we observe by analyzing our simulations, for example in the case $6_{1}$ vs $3{ }_{1} \# 3_{1}$, as shown in Fig. 9(d), or 74 vs $3{ }_{1} \# 4_{1}$ (Fig. 9(h)), where we plot distributions of the rescaled variable $x=l_{1} / N$, for a bet- ter comparison of data with different $N$. The plots show that for increasing $N$ there is a competition between two stable minima of the free energy, corresponding to states in which one of the globules takes the largest part of the backbone length.

When the same type of simulation is performed for a case like $3_{1}$ vs $4_{1}$ (Fig. 9](a)), the scenario changes: $P$ becomes very asymmetric, showing a pronounced, dominant peak for configurations in which the globule with $k=4_{1}$ takes most of the backbone length. The peak for opposite configurations with dominating 3 is considerably depressed and almost disappears in comparison. Panels (b)(c) and (e)-(g) of Fig. 9 show similar cases. The message is very clear: the dominance of only one peak is a consequence of topology and thus should be ascribed to the topological correction.

To check whether these knot-plus-wall simulations yield results compatible with the data from isolated polymers, one may consider combinations of the data that depend exclusively on the expected topological correction. For instance, one can consider the weight "unbalance" (or free energy dif- 
ference)

$$
\begin{aligned}
U_{k_{1}, k_{2}}\left(l_{1} ; N\right) \equiv & \ln \left[P_{k_{1}, k_{2}}\left(l_{1}\right) / P_{k_{1}, k_{2}}\left(N-l_{1}\right)\right] \\
\simeq & \varphi_{k_{1}}\left(l_{1}\right)+\varphi_{k_{2}}\left(N-l_{1}\right) \\
& -\left[\varphi_{k_{1}}\left(N-l_{1}\right)+\varphi_{k_{2}}\left(l_{1}\right)\right] \\
\simeq & \left(M_{k_{1}}-M_{k_{2}}\right) \frac{N-2 l_{1}}{l_{1}\left(N-l_{1}\right)} \\
= & \frac{M_{k_{1}}-M_{k_{2}}}{N} \frac{1-2 x}{x(1-x)}
\end{aligned}
$$

One can note that only a difference $\Delta M=M_{k_{2}}-$ $M_{k_{1}}$ determines this distribution. In Fig. 10 we show a plot of $\ln \left[P_{k_{1}, k_{2}}\left(l_{1}\right) / P_{k_{1}, k_{2}}\left(N-l_{1}\right)\right]$ and of its fit $U_{k_{1}, k_{2}}\left(l_{1} ; N\right)$ (for $T=1 / 0.48, N=500, k_{1}=3_{1}$, $\left.k_{2}=4_{1}\right)$ vs $x=l_{1} / N$. The value $\Delta M \simeq 224$ from the fit is reasonably consistent with the determinations $M_{4_{1}}-M_{3_{1}} \simeq 263$ and $M_{4_{1}}^{\prime}-M_{3_{1}}^{\prime} \simeq 215$ based on the results for knot frequencies (which were summarized in Table 2). This agreement is found for other knot pairs as well, see Table 3 where, for each knot combination and for both $N=500$ and $N=1000$, we list two estimates: $\Delta M_{0}$ from fits of all data and $\Delta M_{1}$ from fits of data with $0.2 \leq x \leq 0.8$. The estimate $\Delta M_{1}$ is introduced because it could be less sensible to knot-independent corrections to scaling, which should be relevant when one of the loops becomes too short. From Table 3 one can see that both estimates are quite consistent with a null value for cases $n_{c 1}=n_{c 2}$ or with values coming from the data for isolated knots.

Thus, the results concerning the competition of globules in a translocation set up give further independent confirmation of the presence of the topological free energy correction and show at the same time its importance for the physics of this process. The outlined intriguing scenario calls for possible fundamental justifications or interpretations of the presence of this correction.

Referring to our ansatz for the globule free energy, Eq. (5), the condition $\frac{\partial}{\partial l_{1}}\left[\varphi_{k_{1}}\left(l_{1}\right)+\varphi_{k_{2}}(N-\right.$ $\left.\left.l_{1}\right)\right]=0$ determining the most probable value of $l_{1}$ becomes independent on $\varphi_{k_{2}}$ if one looks for small values of $l_{1}$ with long $N$ :

$$
\frac{2}{3} \sigma l_{1}^{-1 / 3}+(\alpha-2) l_{1}^{-1}+M_{k_{1}} l_{1}^{-2} \simeq 0
$$

The solution $\overline{l_{1}}$ is indeed a relatively short length.

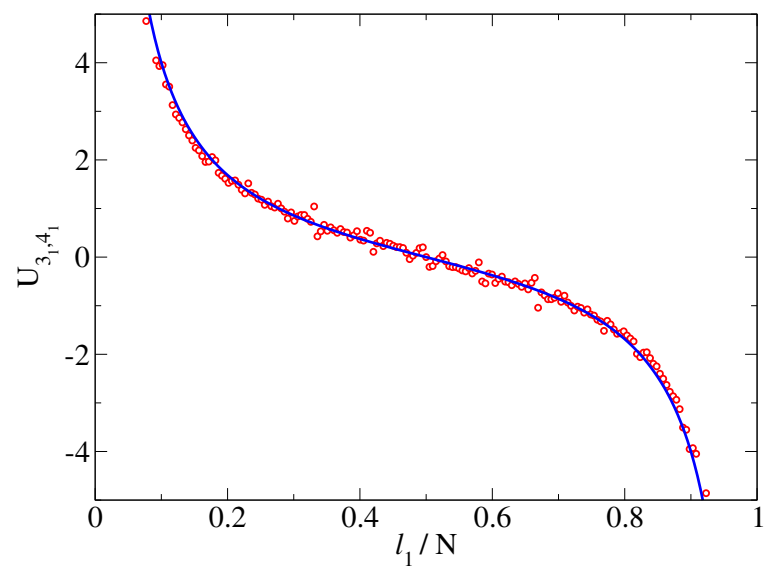

Figure 10: Free energy differences vs. the rescaled loop length for a $33_{1}$ competing with a $4_{1}$ in the configuration of Fig. 6(b) for $N=500$. Points are data in the form $\ln \left[P_{k_{1}, k_{2}}\left(l_{1}\right) / P_{k_{1}, k_{2}}\left(N-l_{1}\right)\right]$ while the solid curve is a plot of $U_{k_{1}, k_{2}}\left(l_{1} ; N\right)$ fitted with Eq. (7) through these data.

This in spite of the fact that we assume still validity of the form in Eq. (5) for the first globule. The last equation gives for $M_{k}$ a leading behavior

$$
M_{k_{1}}(T) \simeq-\frac{2}{3} \sigma(T) \bar{l}_{1}^{5 / 3}-(\alpha-2) \bar{l}_{1}
$$

(we recall that $\sigma<0$ ). The second term on the righthand side is a small correction, and the relation may be approximately inverted to express the typical length $\overline{l_{1}}$ as a function of $M_{k_{1}}$,

$$
\overline{l_{1}}(T) \simeq\left[-\frac{3 M_{k_{1}}(T)}{2 \sigma(T)}\right]^{3 / 5}
$$

Recent results 54 give estimates of $\sigma(T)$ in the range $0.32 \leq 1 / T \leq 0.5$. From these results, at the inverse temperature $1 / T=0.48$ we interpolate $\sigma=-0.96(5)$, which can be used to check that the predictions of (10) are fairly consistent with direct estimates of $\overline{l_{1}}$ from the maxima of $P\left(l_{1}\right)$, see the last two columns of Table 2, There could be a bias of the indirect estimates toward larger values, which might come from knot-independent corrections to scaling. However, the general trend follows closely that of the $\overline{l_{1}}$ of probability maxima.

The relation between the typical length and the corrections to scaling may be extended to include a further relation with the length of ideal knots. For 
Table 3: Fits of $\ln P(l / N) / P(1-l / N)$, for several knots at $1 / T=0.48$ : here $\Delta M_{0}$ is from the fit of the whole data while $\Delta M_{1}$ is from only data with $0.2 \leq l / N \leq 0.8$. Each value is computed for the global ring lengths $N=500$ and $N=1000$. The values $M_{2}-M_{1}$ and $M_{2}^{\prime}-M_{1}^{\prime}$ are determined with the simulations described in the previous section, see Table 2

\begin{tabular}{llllcccc}
\hline & & & & \multicolumn{2}{c}{$N=500$} & \multicolumn{2}{c}{$N=1000$} \\
$k_{1}$ & $k_{2}$ & $M_{2}-M_{1}$ & $M_{2}^{\prime}-M_{1}^{\prime}$ & $\Delta M_{0}$ & $\Delta M_{1}$ & $\Delta M_{0}$ & $\Delta M_{1}$ \\
\hline $3_{1}$ & $4_{1}$ & 263 & $215(36)$ & $224(3)$ & $225(3)$ & $224(6)$ & $189(32)$ \\
$3_{1}$ & $5_{1}$ & 560 & $546(48)$ & $385(2)$ & $408(3)$ & $401(6)$ & $469(30)$ \\
$3_{1}$ & $6_{2}$ & 1018 & $979(56)$ & $735(5)$ & $763(3)$ & $1469(10)$ & $1526(11)$ \\
$4_{1}$ & $5_{1}$ & 297 & $331(42)$ & $163(2)$ & $183(3)$ & $191(3)$ & $253(16)$ \\
$5_{2}$ & $6_{2}$ & 428 & $401(71)$ & $297(3)$ & $298(2)$ & $322(4)$ & $392(10)$ \\
$6_{2}$ & $7_{4}$ & 383 & $570(95)$ & $286(4)$ & $292(3)$ & $308(3)$ & $362(5)$ \\
$6_{2}$ & $3_{1} \# 3_{1}$ & 75 & $112(68)$ & $-53(2)$ & $-48(2)$ & $-53(3)$ & $-9(5)$ \\
$7_{4}$ & $3_{1} \# 4_{1}$ & 24 & $-152(112)$ & $-12(3)$ & $-14(2)$ & $-3(3)$ & $14(4)$ \\
\hline
\end{tabular}
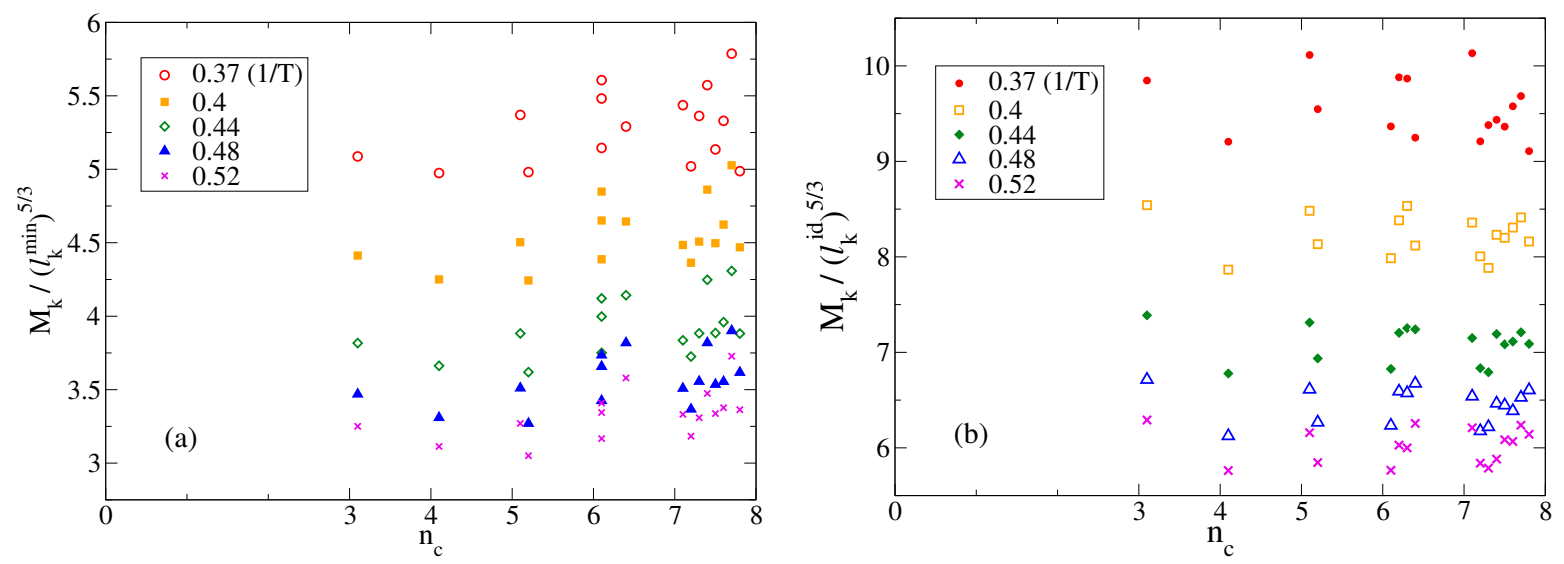

Figure 11: Ratio between correction-to-scaling constants and ideal knot lengths to the power $5 / 3$ (top panel for minimal lengths on the cubic lattice, bottom panel for ideal lengths of knotted curves in three dimensions), for different temperatures. Abscissas are equal to the knot crossing number plus $1 / 10$ of the knot index (with $3_{1} \# 3_{1}$ at 3.4 and $3_{1} \# 4_{1}$ at 7.8 ).

off-lattice knotted flexible yet impenetrable tubes of thickness $D$ and length $L$, the ideal length $l^{\text {id }}$ of a given knot is the smallest ratio $L / D$ one can achieve. ${ }^{3 \mid 10}$ For a lattice knot the "ideal" configurations are those with the minimal number $\left(l^{\mathrm{min}}\right)$ of steps. $\frac{4748}{4}$ The probability maxima discussed above are located at relatively small values of $\overline{l_{1}}$, not too far from $l_{k_{1}}^{\min }$. This suggests that the configurations taken by the loop with $k_{1}$ for $l_{1} \approx \overline{l_{1}}$ could be somehow reminiscent, up to temperature dependent moderate deviations, of the configurations of minimal length, with $\overline{l_{1}} \sim l_{k_{1}}^{\mathrm{min}}$. Evidence of the plausibility of this relation is obtained by reporting our determinations of $M_{k_{1}}$ divided by $\left(l_{k_{1}}^{\min }\right)^{5 / 3}$ in Fig. 11 (a), and divided by $\left(l_{k_{1}}^{\mathrm{id}}\right)^{5 / 3}$ in Fig. 11 (b) (note that the known values suggest that to a first approximation $\left.l^{\mathrm{min}} \propto l^{\mathrm{id}}\right)$. Thus, the existence of the topological correction and its dependence on topology should ultimately follow from the fact that tight knots have a minimal length depending primarily on $n_{c}$. This is but another instance in which the ideal form of knots is in close relation with their physical behavior. 3

We conclude by adding that in principle the terms in Eq. (5) may be complemented with knotindependent corrections to scaling. While we have assessed with nonlinear fits of the distributions $P\left(l_{1}\right)$ that any correction $\sim 1 / l_{1}$ would be present with a prefactor of order unity (as opposed the $M_{k}$ 's, which are at least $\gtrsim 700$ ), there remains to 
understand whether corrections such as $B / l_{1}^{\Delta}$ with $0<\Delta<1$ would play a role. Preliminary results appear to show that $B \approx 100$ if $\Delta=1 / 2$ is assumed. With this magnitude, the corrections would just slightly modify quantitatively but not qualitatively our conclusions above.

\section{Conclusions}

Globular or dense polymers are known as an hard subject in statistical physics, from both theoretical and numerical points of view. With the present contribution we tried to show that they provide an unexpectedly rich context in which to pursue the goals of topological polymer statistics. Indeed, many of the results we obtained are rather unexpected and surprising, in some cases also for their deceptive simplicity. Going deeper into these issues constitutes an open challenge.

When considering rings in the globular phase, any planar projection of the configurations yields an enormous number of crossings,, 57 growing like $N^{4 / 3}$. This huge number of crossings makes extremely difficult the analysis of the type of knot, which requires to sort out the few crossings which are essential characterization of the knot. In spite of this, we showed that the thermodynamics of the globule is driven by the number of these minimal crossings in several ways, and that in some situations $n_{c}$ can become unexpectedly a key parameter for determining the stability of the system.

In first place we clarified the role of topology in determining the spectrum of globular knots in a standard model of collapsed polymer rings. This provides a unique example so far of spectrum in which all knots, prime or composite, enter with comparable frequencies (for swollen rings such balance occurs only within subclasses of knots with the same number of prime components). The remarkable independence of the relative frequencies on temperature suggests that they are universal ratios and poses the question of their link with the knot topological invariants.

Another surprising result is provided by the topological free energy correction and its dependence on the minimal crossing number $n_{c}$. Under the constraint imposed by a slip link, which does not allow trespassing of the knots from one loop to the other, $n_{c}$ appears to control very sharply and exclusively the average share of backbone between the loops. At the same time this average behavior results from an interesting regime of broad fluctuations obeying a form of Taylor scaling. $\underline{58}$

The numerical experiments concerning the translocation of the globule through a repulsive plane with a hole are perhaps the most interesting both within the context of the present investigation, and in the perspective of future applications. Besides providing a strong support to the postulated form of the topological free energy correction, our results indicate how far reaching can be the influence of $n_{c}$ in determining behaviors such as entropic forces driven by topology. Notably, we found that these features are related to the concept of ideal knots, whose signature thus emerges in the statistics of random globular knotted polymers.

Acknowledgement EO acknowledges support from the Italian Ministry of Education grant PRIN 2010 HXAW77.

\section{References}

(1) E. Orlandini, S. G. Whittington, Rev. Mod. Phys. 2007, 79, 611-642.

(2) Deguchi, T.; Tsurusaki, K. Lectures at Knots 96 (World Scientific, Singapore) 1997, 95122.

(3) Stasiak, A., Katrich, V., Kauffmann, L. H., Eds. Ideal Knots; Series on Knots and Everything; World Scientific: Singapore, 1998; Vol. 19.

(4) Dai, L.; van der Maarel, J.; Doyle, P. ACS Macro Lett 2012, 1, 732-736.

(5) Renner, C.; Doyle, P. ACS Macro Lett. 2014, 3, 963-967.

(6) Matthews, R.; Louis, A. A.; Yeomans, J. M. Europhys. Lett. 2010, 89, 20001.

(7) Matthews, R.; Louis, A. A.; Likos, C. N. ACS Macro Lett. 2012, 1, 1352-1356.

(8) Narros, A.; Moreno, A. J.; Likos, C. N. Macromol. 2013, 46, 3654-3666. 
(9) Poier, P.; Likos, C. N.; Matthews, R. Macromol. 2014, 47, 3394-3400.

(10) V. Katritch, J. Bednar, D. Michoud, R. G. Scharein, J. Dubochet, A. Stasiak, Nature 1996, 384, 142-145.

(11) J. Arsuaga, M. Vázquez, P. McGuirk, S. Trigueros, D. W. Sumners, J. Roca, Proc. Natl. Acad. Sci. USA 2005, 102, 9165-9169.

(12) Marenduzzo, D.; Orlandini, E.; Stasiak, A.; Sumners, D. W.; Tubiana, L.; Micheletti, C. Proc. Natl. Acad. Sci. USA 2009, 106, 22269-22274.

(13) Tang, J.; Du, N.; Doyle, P. S. Proc. Natl. Acad. Sci. USA 2011, 108, 16153-16158.

(14) Tubiana, L.; Orlandini, E.; Micheletti, C. Phys. Rev. Lett. 2011, 107, 188302.

(15) Marenduzzo, D.; Micheletti, C.; Orlandini, E.; Sumners, D. W. Proc. Natl. Acad. Sci. USA 2013, 110, 20081-20086.

(16) V. Katritch, W. K. Olson, A. Vologodskii, J. Dubochet, A. Stasiak, Phys. Rev. E 2000, 61, 5545-5549.

(17) O. Farago, Y. Kantor, M. Kardar, Europhys. Lett. 2002, 60, 53-59.

(18) B. Marcone, E. Orlandini, A. L. Stella, F. Zonta, J. Phys. A: Math. Gen. 2005, 38, L15L21.

(19) B. Marcone, E. Orlandini, A. L. Stella, F. Zonta, Phys. Rev. E 2007, 75, 041105-11.

(20) Rawdon, E.; Dobay, A.; Kern, J. C.; Millett, K. C.; Piatek, M.; Plunkett, P.; Stasiak, A. Macromol. 2008, 41, 4444-4451.

(21) Rawdon, E. J.; Kern, J. C.; Piatek, M.; Plunkett, P.; Stasiak, A.; Millett, K. C. Macromol. 2008, 41, 8281-8287.

(22) E. Orlandini, A. L. Stella, C. Vanderzande, Phys. Rev. E 2003, 68, 031804.

(23) A. Hanke, R. Metzler, P. G. Dommersnes, Y. Kantor, M. Kardar, Europ. Phys. J. E 2003, 12, 347-354.
(24) E. Orlandini, A. L. Stella, C. Vanderzande, J. Stat. Phys. 2004, 115, 681-700.

(25) M. Baiesi, E. Orlandini, A. L. Stella, F. Zonta, Phys. Rev. Lett 2011, 106, 258301.

(26) M. Baiesi, E. Orlandini, A. L. Stella, Phys. Rev. Lett. 2007, 99, 058301.

(27) Y. Arai, R. Yasuda, K. Akashi, Y. Harada, H. Miyata, T. Kinosita, H. Itoh, H, Nature 1999, 399, 446-448.

(28) Ayme, J.-F.; Beves, J. E.; Leigh, D. A.; McBurney, R. T.; Rissanen, K.; Schultz, D. Nat. Chem. 2012, 4, 15-20.

(29) A. A. Podtelezhnikov, N. R. Cozzarelli, A. V. Vologodskii, Proc. Natl. Acad. Sci. USA 1999, 96, 12974-12979.

(30) Tkalec, U.; Ravnik, M.; Čopar, S.; Žumer, S.; Muševič, I. Science 2011, 333, 62-65.

(31) Irvine, W. T. M.; Kleckner, D. Nat. Mat. 2014, 13, 229.

(32) M. Baiesi, E. Orlandini, A. L. Stella, J. Stat. Mech. 2010, 10, P06012.

(33) E. Orlandini, M.C. Tesi, E. J. Janse van Rensburg, S. G. Whittington, J. Phys. A: Math. Gen. 1998, 31, 5953-5967.

(34) A. Yao, H. Matsuda, H. Tsukahara, M. K. Shimamura, T. Deguchi, J. Phys. A: Math. Gen. 2001, 34, 7563-7577.

(35) E. J. Janse van Rensburg, A. Rechnitzer, J. Phys. A: Math. Theor. 2011, 44, 162002.

(36) M. Baiesi, E. Orlandini, Phys. Rev. E 2012, 86, 031805.

(37) D. W. Sumners, S. G. Whittington, J. Phys. A: Math. Gen. 1988, 21, 1689-1694.

(38) Y. Diao, N. Pippenger, D. W. Sumners, J. Knot Theory Ramif. 1994, 3, 419-429.

(39) C. Aragao de Carvalho, S. Caracciolo, J. Physique 1983, 44, 323-331.

(40) B. Berg, D. Foester, Phys. Lett. B 1981, 106, 323-326. 
(41) Virnau, P.; Mirny, L. A.; Kardar, M. PLoS Comput Biol 2006, 2, e122.

(42) Sulkowska, J. I.; Sulkowski, P.; Szymczak, P.; Cieplak, M. Proc. Natl. Acad. Sci. USA 2008, 105, 19714-19719.

(43) Potestio, R.; Micheletti, C.; Orland, H. PLoS Comput Biol 2010, 6, e1000864.

(44) J. Dorier, A. Stasiak, Nucl. Acid Res. 2009, 37, 6316-6322.

(45) A. Y. Grosberg, Polymer Science 2012, 54, $1-10$.

(46) E. J. Janse van Rensburg and S. D. Promislow, J. Knot Theory Ramif. 1995, 4, 115.

(47) Y. Diao, J. Knot Theo. Ramif. 1993, 2, 413425.

(48) Janse van Rensburg, E. J.; Rechnitzer, A. J. Stat. Mech. 2011, P09008.

(49) Tesi, M.; Janse van Rensburg, E. J.; Orlandini, E.; Whittington, S. G. J. Phys. A: Math. Theor. 1996, 29, 2451-2463.

(50) Grassberger, P. Phys. Rev. E 1997, 56, 36823693.

(51) Grassberger, P.; Hsu, H. P. Phys. Rev. E 2002, 65, 031807.

(52) N. Madras and G. Slade, The Self-Avoiding Walk; Birkhäuser: Boston, 1993.

(53) J. Hoste and M. Thistlethwaite, http://www.math.utk.edu/ morwen/knotscape.html.

(54) Baiesi, M.; Orlandini, E. Phys. Rev. E 2014, 89, 062601.

(55) Owczarek, A. L.; Prellberg, T.; Brak, R. Phys. Rev. Lett. 1993, 70, 951-953.

(56) R. C. Lua, A. L. Borowinskiy, A. Y. Grosberg, Polymer 2004, 45, 717.

(57) Grassberger, P. J. Phys. A: Math. Gen. 2001, 34, 9959.

(58) Eisler, Z.; Bartos, I.; Kertész, J. Adv. Phys. 2008, 57, 89-142. 